\title{
Lesiones de hombro atribuibles a la aplicación de la vacuna contra la COVID-19
}

\author{
Daniel Moya, ${ }^{\star}$ Diego Gómez, ${ }^{\star}$ Rufino C. Ruíz,** Marcela Balzarini, ${ }^{* * *}$ Laura Muñoz González \\ "Servicio de Ortopedia y Traumatología, Hospital Británico de Buenos Aires, Ciudad Autónoma de Buenos Aires, Argentina \\ **Servicio de Ortopedia y Traumatología, Sanatorio Dupuytren, Ciudad Autónoma de Buenos Aires, Argentina \\ ${ }^{* * *}$ Unidad Febril de Urgencia, Hospital "Bernardino Rivadavia", Ciudad Autónoma de Buenos Aires, Argentina \\ "Servicio de Fisiatría, Complejo Asistencial Zamora, Castilla y León, España
}

\begin{abstract}
RESUMEN
Las lesiones de hombro atribuibles a la vacunación son aquellos efectos adversos secundarios a una inoculación. Su causa más frecuente ha sido la vacunación contra la gripe, pero la actual campaña masiva por la pandemia de SARS-CoV-2 determina que los especialistas conozcan este cuadro para un mejor diagnóstico y tratamiento. Estas lesiones tienen una evolución tórpida y prolongada. Deben notificarse a las agencias de control sanitario. Su tratamiento se basa en la administración temprana de corticoides y la rehabilitación.
\end{abstract}

Palabras clave: COVID-19; bursitis subacromial; vacuna; dolor de hombro.

Nivel de Evidencia: $\mathrm{V}$

\section{Shoulder Injuries Related to COVID-19 Vaccination}

\section{ABSTRACT}

Shoulder injury attributable to vaccination (SIRVA) is an adverse event secondary to inoculation. Its most frequent cause has been vaccination against influenza, but the current massive campaign against the SARS-CoV-2 pandemic makes it an injury whose knowledge is of importance for specialists. It includes different structural lesions and has a torpid and prolonged evolution. SIRVAs must be reported to the sanitary control agencies. Their treatment is based on the use of corticosteroids and rehabilitation.

Key words: COVID-19; bursitis; vaccine; shoulder pain.

Level of Evidence: $\mathrm{V}$

\section{INTRODUCCIÓN}

La emergencia mundial generada por la irrupción de la pandemia de SARS-CoV-2 ha determinado la necesidad de una campaña masiva de vacunación con el fin de lograr la inmunización colectiva rápidamente. Esto conlleva la aplicación diaria de millones de vacunas en todos los rincones del planeta. ${ }^{1}$

El lugar utilizado para la inoculación es el músculo deltoides por tratarse de un área segura, que permite una buena respuesta inmunológica con bajos efectos adversos, sin dificultar la deambulación y es un área de fácil exposición para el paciente. ${ }^{2,3}$ Sin embargo, no está libre de complicaciones. El efecto adverso comunicado con más frecuencia, cualquiera sea el tipo de vacuna aplicada en el hombro, es el dolor en la zona de inoculación. ${ }^{4-9}$ También puede haber enrojecimiento, picazón, edema, dolor a la palpación, induración y hematoma. ${ }^{4-12}$ Todos estos síntomas suelen ser leves y transitorios, desaparecen en dos o tres días luego de la vacunación. ${ }^{12}$

En un porcentaje menor de casos, se produce una sintomatología local severa y persistente que incluye dolor intenso, pérdida de la movilidad activa y pasiva e incapacidad funcional. La sigla en inglés que identifica a esta situación es SIRVA (Shoulder Injury Related to Vaccine Administration), término con implicancias medicolegales que fue acuñado por Atanasoff, en 2010. ${ }^{4}$ No hemos encontrado reportes en español relacionados con este cuadro. Lo hemos traducido como "Lesiones de Hombro Atribuibles a la Vacunación" (LHAV).

Recibido el 21-8-2021. Aceptado luego de la evaluación el 23-8-2021 • Dr. DANIEL MOYA • drdanielmoya @ gmail.com

Cómo citar este artículo: Moya D, Gómez D, Ruíz RC, Balzarini M, Muñoz González L. Lesiones de hombro atribuibles a la aplicación de la vacuna contra la COVID-19. Rev Asoc Argent Ortop Traumatol 2021;86(6):842-849. https://doi.org/10.15417/issn.1852-7434.2021.86.6.1421 
El objetivo de esta presentación es brindar información al traumatólogo general para poder diagnosticar, reportar y tratar adecuadamente estos casos.

\section{Definición}

Sería un error considerar que todo cuadro doloroso localizado en el hombro luego de la aplicación de una vacuna sea atribuible a esta. Un alto porcentaje de las personas que reciben la vacuna inyectable contra la gripe informan sensibilidad, hinchazón y dolor en el lugar de aplicación, ${ }^{4}$ pero esto no justifica la calificación de LHAV.

Existe una definición muy precisa que incluye una serie de condiciones para poder considerar a una lesión severa como atribuible a la aplicación de una vacuna: ${ }^{13,14}$

1. El cuadro de dolor debe comenzar dentro de las $48 \mathrm{~h}$ posteriores a la inoculación.

2. La sintomatología debe durar, al menos, siete días desde su comienzo.

3. El cuadro incluye la pérdida de la movilidad activa que también se puede acompañar de disminución de la movilidad pasiva.

4. La sintomatología previa inmediata a la inoculación pone en duda la existencia de un evento atribuible al acto de la vacunación.

Las LHAV se presentan más comúnmente con bursitis subacromial y subdeltoidea, ${ }^{4-6,8,9-15}$ y lesiones del manguito rotador. ${ }^{4,5,8,13,15}$ En cuanto a los tendones más afectados, se han comunicado lesiones en el supraespinoso, subescapular, infraespinoso y redondo menor. ${ }^{13,14}$ Szari y cols. ${ }^{10}$ describieron un caso de LHAV con imágenes por resonancia magnética de tendinopatía en los cuatro tendones del manguito rotador y posible tendinopatía calcificante del redondo menor.

Se han reportado tendinopatía bicipital, ${ }^{4}$ sinovitis y derrame glenohumeral, ${ }^{14-16}$ cuadros de capsulitis adhesiva, ${ }^{5,8,11,16}$ miositis, ${ }^{16}$ formación de abscesos estériles intramusculares, ${ }^{15}$ osteítis, ${ }^{16}$ acumulación de líquido en el deltoides ${ }^{16}$ (Figura 1) y en el manguito rotador, ${ }^{16}$ artritis erosiva, ${ }^{16}$ artritis séptica, ${ }^{8}$ osteomielitis ${ }^{17}$ y osteonecrosis ${ }^{18}$.

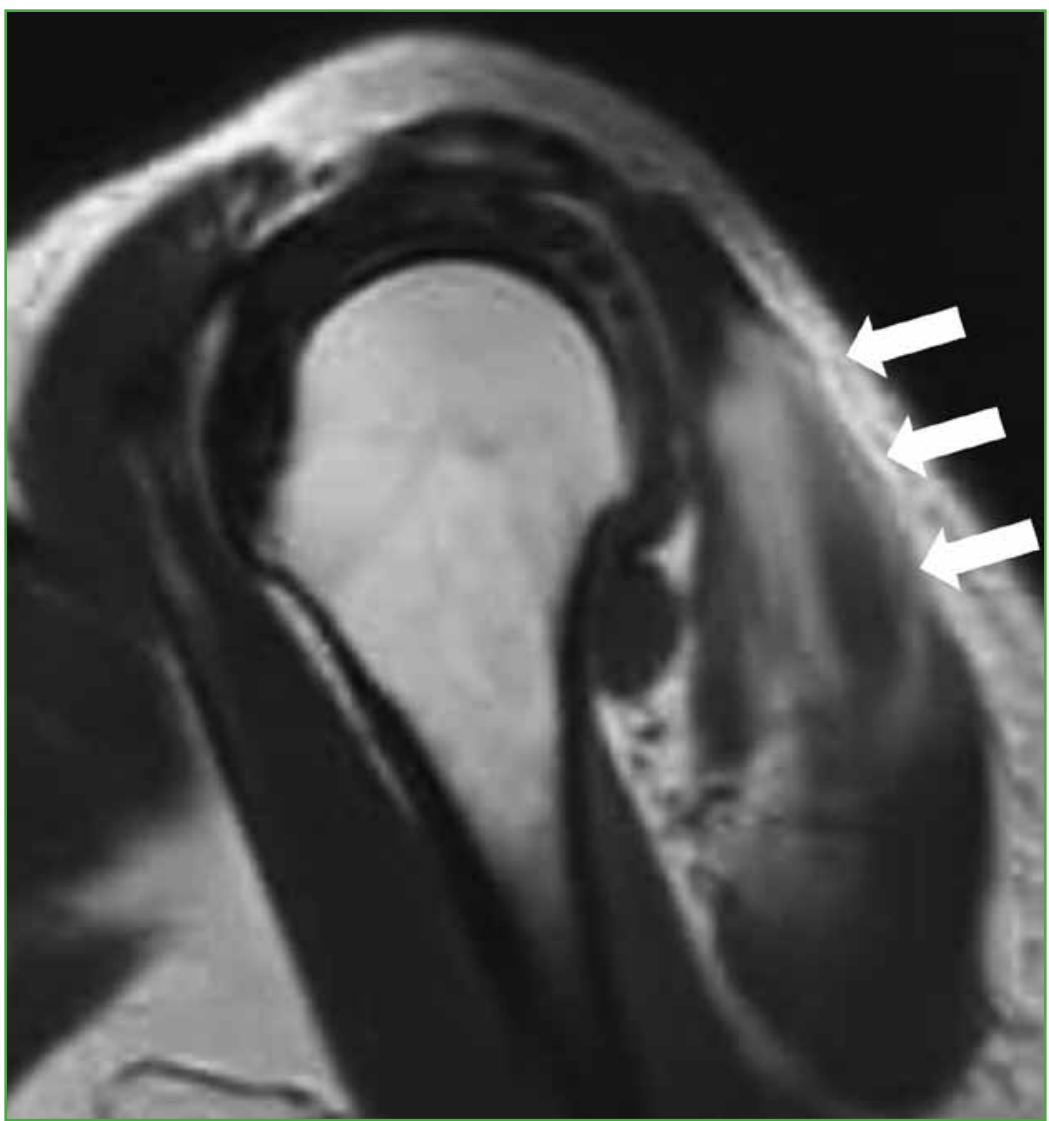

Figura 1. Imagen líquida en el espesor del músculo deltoides después de la aplicación de la vacuna contra la COVID-19. 
También se han publicado informes de lesiones nerviosas que incluyen la rama anterior del nervio axilar, radiculopatía de C6 y parálisis radial completa. ${ }^{19,20}$ Se han comunicado casos de síndrome de Parsonage-Turner secundarios a la vacunación. ${ }^{21}$

La experiencia es más limitada con la vacunación contra la COVID-19, porque su implementación es reciente. Hasta el momento, se han informado dos casos de bursitis subacromial, ${ }^{12,15}$ un caso de artritis séptica ${ }^{8}$ y cuatro casos de plexitis braquial ${ }^{21}$. Cabe resaltar que el caso publicado por Chuaychoosakoon y cols. ${ }^{12}$ es discutible porque, en las radiografías, se aprecia una avanzada artrosis glenohumeral previa.

\section{Epidemiología}

No se conoce la tasa exacta de complicaciones locales severas secundarias a la vacunación en el área del hombro. Indudablemente existe un reporte inferior de efectos adversos. ${ }^{5,10}$ Uno de los factores que contribuye a esto es que el sistema, en la mayoría de los países, se basa en la notificación voluntaria de sospechas de reacciones adversas por parte de los profesionales de la salud y de los pacientes, por lo que es difícil medir la incidencia real.

En los Estados Unidos, existe el National Vaccine Injury Compensation Program (VICP) que, desde 2010, recibe los reclamos relacionados con las lesiones presuntamente provocadas por la vacunación. Según el informe del Vaccine Adverse Event Reporting System (VAERS) de ese país, entre 2010 y 2016, hubo 1006 informes de disfunción del hombro después de la aplicación de la vacuna antigripal inactivada en comparación con un estimado de 130 millones de dosis de esta vacuna administrada cada temporada de gripe en los EE.UU. ${ }^{10}$

Si bien el porcentaje es bajísimo, las denuncias se han incrementado progresivamente. En 2011, se presentaron siete reclamos ante el Programa VICP, mientras que, en 2016, fueron $446 .^{13}$

La bibliografía revela que la mayoría de los casos ocurren en mujeres. ${ }^{4,5} \mathrm{Si}$ bien los reportes corresponden a un amplio rango etario (de 21 meses a 90 años), lo más frecuente es que se produzca en la edad media de la vida, con un promedio de alrededor de 50 años. ${ }^{5}$ El motivo más frecuente es la vacunación contra la gripe, ${ }^{4,5}$ pero puede ocurrir después de cualquier tipo de vacuna aplicada en el hombro.

Casi la mitad de las personas con lesiones en el hombro informaron que les habían aplicado la vacuna demasiado alto en el brazo. ${ }^{4} \mathrm{El}$ dolor suele aparecer en las primeras $48 \mathrm{~h}$ posteriores a la vacunación. ${ }^{4,14,19}$

\section{Mecanismo de la lesión}

En la mayoría de los casos, el cuadro está generado por una técnica inadecuada de inoculación. ${ }^{3,8,12-15}$ Se han descrito detalladamente las áreas seguras de inoculación, el largo adecuado de las agujas según las características del paciente y otros detalles técnicos.

La técnica puede fallar a causa de una inoculación muy alta que provoca un compromiso de la bursa subacromiosubdeltoidea, o muy baja con posible compromiso neurológico, o muy profunda con agresión del periostio o la articulación, ${ }^{13}$ o muy superficial depositando la vacuna en el tejido celular subcutáneo que no está suficientemente vascularizado como para facilitar una adecuada respuesta inmune y se asocia con un aumento de las reacciones adversas locales. $^{17}$

Más allá de esto, la lesión originada por la vacunación suele ser mayor de lo que cabría esperar de un simple traumatismo con una aguja., ${ }^{45}$ Cuando se inocula una vacuna, se produce una reacción antígeno-anticuerpo en el músculo que genera molestias, pero en general, son transitorias y moderadas. Cuando la solución de la vacuna es inadvertidamente inyectada en un espacio sinovial, como los del hombro, los anticuerpos de infecciones o vacunaciones previas pueden conducir a una reacción inflamatoria mucho más prolongada. ${ }^{4,15}$ Aunque la COVID-19 es una enfermedad nueva y la población está recibiendo vacunas por primera vez, es poco probable que un adulto no haya estado expuesto a un adenovirus en el pasado.

Thompson y Ensrud ${ }^{11}$ publicaron un caso de capsulitis adhesiva de ambos hombros después de la aplicación de la vacuna antigripal en uno de ellos, esto sugiere una respuesta inmunomediada.

Aunque los estudios mencionados avalan la posibilidad de una respuesta inmunitaria, aún no se ha llevado a cabo un estudio clínico definitivo que respalde esta teoría.

Por último, los compuestos de aluminio en el adyuvante de algunas vacunas pueden haber contribuido también a provocar reacciones inflamatorias intensas. ${ }^{5}$ 


\section{Cuadro clínico}

Típicamente los pacientes se presentan con un cuadro de dolor intenso y disminución del rango de movilidad después de la inoculación de la vacuna. En muchos casos, el dolor comienza en el acto, sobre todo, cuando la aguja ingresó en la bursa subacromial o generó una lesión en los nervios periféricos. En esta última situación, el cuadro comienza con dolor urente en el mismo acto de la inoculación. En casos de sepsis, podrá haber fiebre e incluso escalofríos. ${ }^{8}$

El dolor es de moderado a intenso y se localiza en la zona deltoidea con propagación al brazo. El mantenimiento en el tiempo llevará al compromiso cervicodorsal.

En la etapa aguda, es importante evaluar y documentar el área de ingreso de la aguja. Se podrá observar eritema, tumefacción y hematoma. En etapas crónicas, esto desaparece y prevalece la atrofia muscular. La palpación de la región deltoidea suele ser dolorosa.

La movilidad activa está severamente limitada por el dolor y, con el paso del tiempo, puede instalarse un cuadro de capsulitis adhesiva con pérdida de la movilidad pasiva.

Siempre deben evaluarse la columna cervical y el estado cardiovascular del paciente.

\section{Exámenes complementarios}

Las imágenes de estudios complementarios variarán de acuerdo con el tipo de lesión. Las radiografías son inespecíficas y, en general, no proporcionan información diagnóstica útil.

Los hallazgos iniciales denotan cambios inflamatorios en partes blandas. Es frecuente detectar un aumento del líquido dentro de la bursa subacromial/subdeltoidea en la ecografía y la resonancia magnética (Figura 2). Salmon y cols. ${ }^{22}$ compararon estas imágenes iniciales con las diferidas a los cinco meses y observaron una regresión del derrame articular y una disminución de la bursitis, por lo que estos estudios pueden no ser muy demostrativos en cuadros crónicos..$^{14}$

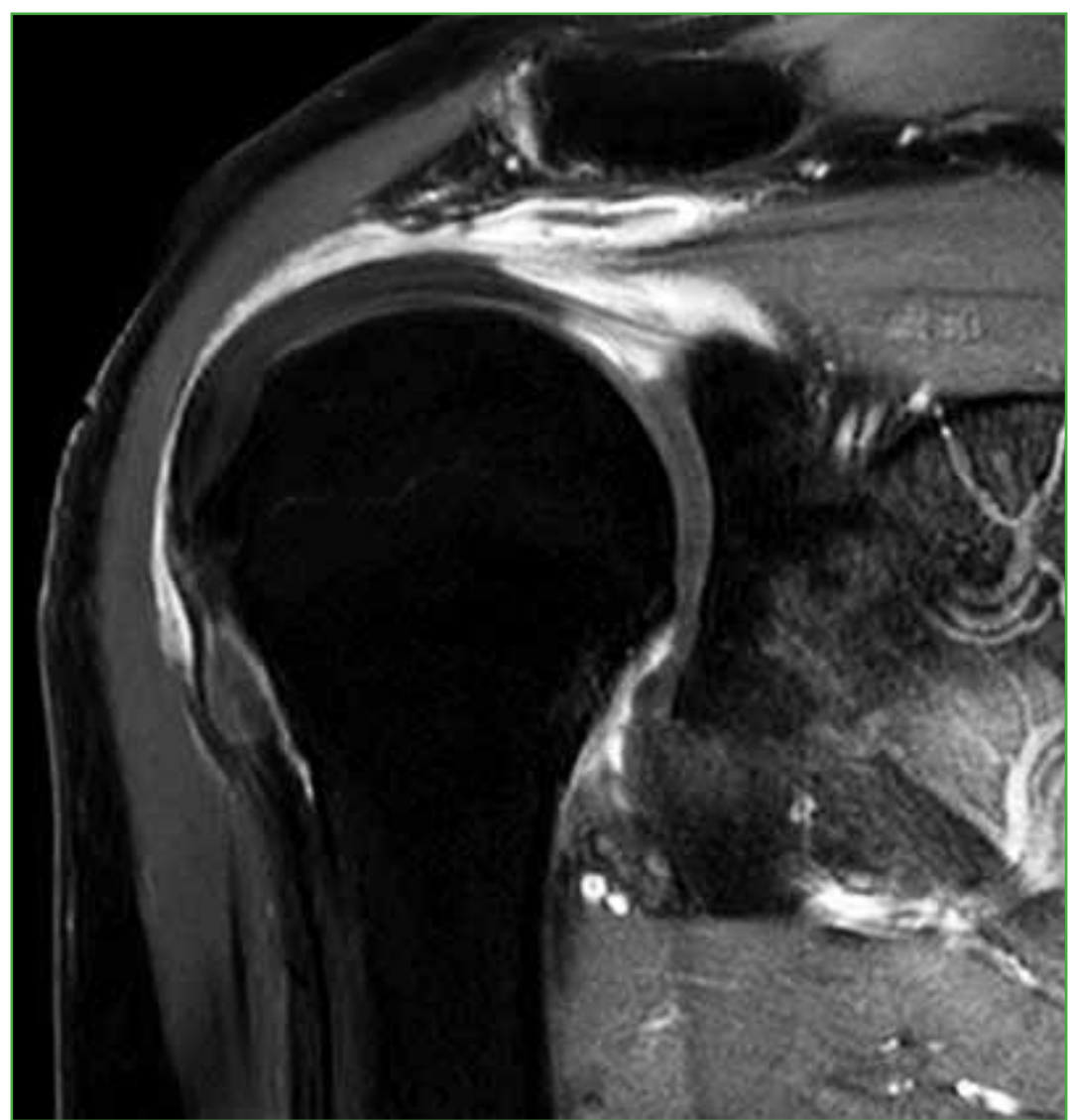

Figura 2. Distensión de la bursa subacromial-subdeltoidea después de la aplicación de la vacuna contra la COVID-19. 
La miositis relacionada con la vacuna se manifiesta como un edema focal o difuso dentro del músculo en el lugar de la inyección, más comúnmente en el deltoides. ${ }^{16}$

Es posible detectar signos de tendinopatía o incluso de rotura parcial y de espesor completo del manguito rotador, pero suelen ser lesiones previas.

Los cambios óseos incluyen edema medular focal, típicamente en la cara lateral del húmero proximal. ${ }^{23}$ También se han descrito lesiones corticales erosivas.

Se publicó un caso de osteonecrosis que se manifestó con hallazgos típicos en los estudios por imágenes, como hiperemia, edema de médula ósea y colapso de la cabeza humeral..$^{18}$ Asimismo, se observaron señales focales de la médula ósea dentro de la cabeza humeral, probablemente por la inyección de la sustancia de la vacuna directamente en las estructuras óseas que podría conducir a una osteítis focal. ${ }^{23}$

Siempre es una buena idea solicitar análisis de laboratorio, como hemograma, eritrosedimentación y reactantes de fase aguda, cuando el paciente consulta por primera vez.

Si existen dudas con respecto al compromiso neurológico periférico, se debe tener en cuenta que debe transcurrir como mínimo un mes para que un estudio electrofisiológico sea significativo. Si hay signos compatibles con el síndrome de Parsonage-Turner, es útil solicitar una resonancia del plexo braquial con medio de contraste y sin él.

\section{Diagnóstico diferencial}

Se han descrito más de 90 causas de dolor de hombro. Se debe sospechar la posibilidad de LHAV en un paciente que se presenta con dolor agudo de hombro después de la administración de una vacuna en la región deltoidea.

Si el paciente tiene una enfermedad confirmada antes del evento, síntomas previos o imágenes de lesiones crónicas, estos hallazgos pondrán en duda que sufra una LHAV, pero no la descartan.

Lo primero que hay que descartar es un cuadro séptico. Distinguir la bursitis infecciosa de la relacionada con la vacuna es particularmente difícil en las imágenes por sí solas. Si bien es muy probable que las radiografías del hombro tengan una apariencia normal, puede haber irregularidad cortical del húmero. ${ }^{23}$ En la resonancia magnética, la bursa llena de líquido aparecerá hiperintensa en la secuencia T2. En la ecografía, se observará como una estructura anecoica llena de líquido rodeada de paredes hiperecoicas. ${ }^{23}$ En estos casos, la historia clínica y los resultados de los análisis de laboratorio son los elementos más útiles para distinguir estas etiologías.

El diagnóstico de una lesión secundaria a la inoculación de una vacuna en la región deltoidea puede parecer muy evidente y simple, pero no siempre el paciente es evaluado inicialmente por un traumatólogo. Se ha publicado un caso de un paciente con diagnóstico de síndrome de Parsonage-Turner, tratado con gabapentina por un neurólogo, aunque, en realidad, se trataba de una osteomielitis del extremo proximal del húmero. ${ }^{17}$ Uno de nuestros pacientes fue tratado por un neurólogo con pregabalina durante cuatro meses, sin respuesta clínica; en realidad, se trataba de un cuadro de bursitis secundaria a una inoculación practicada inadecuadamente.

La LHAV es una situación que puede determinar una larga lista de trastornos musculoesqueléticos que ya hemos mencionado; por este motivo, consideramos que la participación de un especialista en Ortopedia y Traumatología es esencial para su manejo diagnóstico y terapéutico.

Como se sabe la prevalencia de lesiones del manguito rotador es altísima en la población asintomática; por lo tanto, su sola presencia en un cuadro de LHAV no implica necesariamente que sea producto de la inoculación.

\section{Tratamiento}

En la actualidad, no existe consenso en la bibliografía sobre cómo manejar estas lesiones relacionadas con la vacunación. Tras descartar una infección y considerando que se trata de un proceso inflamatorio agudo que puede afectar distintas estructuras del hombro, lo ideal es comenzar inmediatamente con la administración de corticoides.

Atanasoff y cols. ${ }^{4}$ comunicaron que más de la mitad de una serie de 13 pacientes con LHAV requirieron, al menos, una inyección de corticoides en algún momento.

Se ha propuesto la aplicación de corticoides en el espacio subacromial, pero no hay evidencia científica que la respalde en este cuadro. ${ }^{24}$ Teniendo en cuenta que se trata de una situación de intenso dolor en el hombro, sumar otra agresión mecánica en la zona no es una buena idea. Según nuestra experiencia, el uso de corticoides de depósito en la región glútea tiene el mismo efecto analgésico que el aplicado en el espacio subacromial, con muchos menos riesgos.

En los casos de síndrome de Parsonage-Turner, además del uso inicial de corticoides, se ha propuesto la administración de inmunoglobulinas basándose en la hipótesis neuroalérgica del síndrome. En pacientes con lesiones neurológicas periféricas, es también habitual administrar complejos vitamínicos B. 
La rehabilitación con ejercicios suaves que incluya un programa domiciliario de ejercicios, es esencial y aconsejada por todos los autores. ${ }^{4,14} \mathrm{Su}$ implementación temprana y progresiva contribuye a controlar el dolor y a prevenir la instalación de cuadros de hombro congelado.

En casos crónicos, puede estar indicado administrar agentes miorrelajantes cuando hay compromiso cervicodorsal. En cuadros que no responden al tratamiento conservador, se ha indicado la cirugía. ${ }^{4,15}$ Llamativamente, en la serie de Atanasoff y cols., ${ }^{4}$ el $31 \%$ de los pacientes requirió una intervención quirúrgica, y la mitad de ellos necesitó una segunda cirugía.

\section{Pronóstico}

La recuperación de estos cuadros no es fácil ni rápida. La mejoría definitiva puede llevar desde semanas hasta años. ${ }^{4}$ En la primera serie publicada que incluía 13 pacientes, ${ }^{4}$ menos de un tercio se recuperó por completo. La mayoría sufrió síntomas persistentes, como dolor y limitación del rango de movilidad a largo plazo, y todos tuvieron síntomas durante, al menos, seis meses. ${ }^{4}$

\section{Reporte sanitario}

La Dirección de Control de Enfermedades Inmunoprevenibles, dependiente del Ministerio de Salud, en el documento de diciembre de 2020 llamado "Lineamientos Técnicos para la Campaña Nacional de Vacunación contra la COVID-19", define el concepto de Evento Adverso Supuestamente Atribuible a la Vacunación e Inmunización (ESAVI). Se considera ESAVI a "cualquier situación de salud no esperada (signo no favorable o no intencionado, hallazgo anormal de laboratorio, síntoma o enfermedad) que ocurre posterior a la vacunación y que no necesariamente tiene una relación causal con la vacunación o con el producto biológico".

Un ESAVI grave es "todo aquel evento que deriva en hospitalización o la prolonga, resulta en discapacidad significativa o persistente, anomalía congénita o fallecimiento. Los eventos graves deben notificarse de manera obligatoria al sistema de vigilancia en forma inmediata". Consideramos que la LHAV es un ESAVI grave por implicar, en muchos casos, una incapacidad significativa y persistente.

Todos los ESAVI deben ser notificados. También deben notificarse todos los "errores de la vacunación" que pueden o no haber generado un efecto adverso, por ejemplo, errores durante la aplicación, utilización de diluyentes no apropiados, agujas incorrectas, vías de aplicación equivocadas, la aplicación de sobredosis. Si, por error, se hubiera administrado la vacuna por otra vía distinta de la intramuscular, la dosis debe considerarse no válida y notificarse como ESAVI.

Para informar un ESAVI se debe completar una ficha de notificación que se puede obtener en internet ${ }^{25} \mathrm{y}$ enviarla a la autoridad competente de acuerdo con cada jurisdicción. En el caso de la Ciudad Autónoma de Buenos Aires al correo electrónico: reportesesavicaba@gmail.com. Esa información se comunicará finalmente a la plataforma SIISA (Sistema Integrado de Información Sanitaria Argentino).

\section{Consideraciones finales}

Las LHAV son poco frecuentes, pero cuando ocurren, su evolución es tórpida y su manejo, complicado. La posibilidad de LHAV no supera las enormes ventajas de la vacunación. Se debe sospechar este cuadro en todas las personas sin antecedentes de dolor o disfunción del hombro que experimenten dolor repentino y reducción del rango de movilidad después de una inoculación en el área deltoidea.

El mejor proceder en esto casos incluye: 1) el diagnóstico temprano, 2) descartar una infección y lesiones neurológicas, 3) detectar qué estructuras musculoesqueléticas están comprometidas, 4) el tratamiento inicial con corticoides, 5) la rehabilitación suave que incluya un programa domiciliario de ejercicios, 6) la notificación obligatoria a las autoridades sanitarias. 
Conflicto de intereses: Los autores no declaran conflictos de intereses.

ORCID de M. Balzarini: https://orcid.org/0000-0002-8306-5517 ORCID de L. Muñoz González: https://orcid.org/0000-0002-5323-3276

\section{BIBLIOGRAFÍA}

1. Our World in Data. Coronavirus (COVID-19) Vaccinations. [Consulta: 17 de agosto, 2021]. Disponible en: https://ourworldindata.org/covid-vaccinations

2. Shaw FE Jr, Guess HA, Roets JM, Mohr FE, Coleman PJ, Mandel EJ, et al. Effect of anatomic injection site, age and smoking on the immune response to hepatitis B vaccination. Vaccine 1989;7(5):425-30. https://doi.org/10.1016/0264-410x(89)90157-6

3. General Recommendations on Immunization. Recommendations of the Advisory Committee on Immunization Practices. (ACIP) Center for Disease Control and Prevention. [Consulta: 21 de agosto, 2021]. Disponible en: https://www.cdc.gov/mmwr/preview/mmwrhtml/rr5515a1.htm

4. Atanasoff S, Ryan T, Lightfoot R, Johann-Liang R. Shoulder injury related to vaccine administration (SIRVA). Vaccine 2010;28(51):8049-52. https://doi.org/10.1016/j.vaccine.2010.10.005

5. Martín Arias LH, Sanz Fadrique R, Sáinz Gil M, Salgueiro-Vazquez ME. Risk of bursitis and other injuries and dysfunctions of the shoulder following vaccinations. Vaccine 2017;35(37):4870-6. https://doi.org/10.1016/j.vaccine.2017.07.055

6. Cook IF. Subdeltoid/subacromial bursitis associated with influenza vaccination. Hum Vaccin Immunother 2014;10(3):605-6. https://doi.org/:10.4161/hv.27232

7. Kaur RJ, Dutta S, Bhardwaj P, Charan J, Dhingra S, Mitra P, et al. Adverse events reported from COVID-19 vaccine trials: A systematic review. Indian J Clin Biochem 2021;1-13. https://doi.org/10.1007/s12291-021-00968-z

8. Massel DH, Haziza S, Rivera S, Mohile N, Subhawong TK, Hernandez VH. Septic arthritis of the shoulder after SARS-CoV-2 Pfizer vaccination: A case report. JBJS Case Connect 2021;11(3). https://doi.org/10.2106/JBJS.CC.21.00090

9. Hexter AT, Gee E,Sandher D. Management of glenohumeral synovitis secondary to influenza vaccination. Shoulder Elbow 2015;7(2):100-3. https://doi.org/10.1177/1758573214560258

10. Szari S, Belgard A, Adams K, Freiler J. Shoulder injury related to vaccine administration: A rare reaction. Fed Pract 2019;36(8):380-4. PMID: 31456630

11. Thompson AR, Ensrud ER. Bilateral adhesive capsulitis following influenza vaccination: A case report. Clin Case Rep 2020;8(11):2155-7. https://doi.org/10.1002/ccr3.3072

12. Chuaychoosakoon C, Parinyakhup W, Tanutit P, Maliwankul K, Klabklay P. Shoulder injury related to Sinovac COVID-19 vaccine: A case report. Ann Med Surg (Lond) 2021;68:102622. https://doi.org/10.1016/j.amsu.2021.102622

13. Natanzi N, Hebroni F, Bodor M. Teres minor injury related to vaccine administration. Radiol Case Rep 2020;14;15(5):552-5. https://doi.org/10.1016/j.radcr.2020.02.009

14. Cagle PJ Jr. Shoulder injury after vaccination: A systematic review. Rev Bras Ortop (Sao Paulo) 2021;56(3):299306. https://doi.org/10.1055/s-0040-1719086

15. Cantarelli Rodrigues T, Hidalgo PF, Skaf AY, Serfaty A. Subacromial-subdeltoid bursitis following COVID-19 vaccination: a case of shoulder injury related to vaccine administration (SIRVA). Skeletal Radiol 2021;4:1-5. https//doi.org/10.1007/s00256-021-03803-x

16. Okur G, Chaney KA, Lomasney LM. Magnetic resonance imaging of abnormal shoulder pain following influenza vaccination. Skeletal Radiol 2014;43(9):1325-31. https://doi.org/10.1007/s00256-014-1875-9

17. Smith SS, Lee Y, Wang L. Adolescent with osteomyelitis after intramuscular administration of a vaccine: A case report. J Am Pharm Assoc (2003) 2020;60(6):e357-e360. https://doi.org/10.1016/j.japh.2020.03.015

18. Kuether G, Dietrich B, Smith T, Peter C, Gruessner S. Atraumatic osteonecrosis of the humeral head after influenza A-(H1N1) v-2009 vaccination. Vaccine 2016;29(40):6830-3. https://doi.org/10.1016/j.vaccine.2011.07.052 
19. Meirelles H, Filho GRM. Lesão do nervo axillar causada pela injeção intramuscular no deltoid: relato de caso/ Axillary nerve Injury caused by deltoid muscle intramuscular injection: case report. Rev Bras Ortop 2004;39:615-9. Disponible en: https://cdn.publisher.gn1.link/rbo.org.br/pdf/39-9/2004_out_07.pdf

20. Ling CM, Loong SC. Injection injury of the radial nerve. Injury 1976;8(1):60-2. https://doi.org/10.1016/0020-1383(76)90012-7

21. Queler SC, Towbin AJ, Milani C, Whang J, Sneag DB. Parsonage-Turner syndrome following COVID-19 vaccination: MR neurography. Radiology 2021;211374. https://doi.org/10.1148/radiol.2021211374

22. Salmon JH, Geoffroy M, Eschard JP, Ohl X. Bone erosion and subacromial bursitis caused by diphtheria-tetanuspoliomyelitis vaccine. Vaccine 2015;33(46):6152-5. https://doi.org/10.1016/j.vaccine.2015.09.090

23. Wright A, Patel R, Motamedi D. Influenza vaccine-related subacromial/subdeltoid bursitis: A case report. $J$ Radiol Case Rep 2019;13(6):24-31. https://doi.org/10.3941/jrcr.v13i6.3656

24. Macomb CV, Evans MO, Dockstader JE, Montgomery JR, Beakes DE. Treating SIRVA early with corticosteroid injections: A case series. Mil Med 2020;185(1-2):e298-e300. Erratum in: Mil Med. 2020;185(1-2):e325. https://doi.org/10.1093/milmed/usz269

25. https://bancos.salud.gob.ar/sites/default/files/2020-12/ficha-notificacion-esavi.pdf. [Consulta: 21 de agosto, 2021.] 